\title{
Conhecimentos de uma equipe multidisciplinar de um Centro de Atenção Psicossocial Infanto-Juvenil sobre o transtorno espectro autista
}

\author{
Knowledge of a multidisciplinar team of a Child Psychosocial Care Center about autistic \\ spectrum disorder
}
Conocimiento de un equipo multidisciplinario de un Centro de Atención Psicosocial Infantil sobre el transtorno del espectro autista

Mayanny da Silva Lima ${ }^{1}$, Gilma Sannyelle Silva Rocha ${ }^{1 *}$, Najra Danny Pereira Lima ${ }^{1}$, Marcus Vinicius da Rocha Santos da Silva ${ }^{2}$, Valeria Silva Carvalho ${ }^{1}$, Mychelle Maria Santos de Oliveira', Maria Camila da Silva ${ }^{1}$, Thalia Costa Medeiros ${ }^{1}$, Thais Costa Medeiros ${ }^{3}$.

\section{RESUMO}

Objetivo: Averiguar os conhecimentos de uma equipe multidisciplinar de um Centro de Atenção Psicossocial Infanto-Juvenil sobre o Transtorno do Espectro Autista e identificar a relação das características do Transtorno com o Brincar da criança. Métodos: Tratou-se de uma pesquisa de caráter descritiva, exploratório, com abordagem qualitativa. O cenário desta investigação foi uma cidade do estado do Maranhão, utilizou-se como campo de pesquisa o Centro de Atenção Psicossocial infanto-juvenil (CAPSIJ), a amostra foi composta por membros da equipe multidisciplinar do referido CAPS. Foi utilizado como material de coleta de dados um questionário com perguntas abertas. Resultados: Os participantes do estudo, em geral, possuem conhecimento superficial sobre o TEA, e, em alguns casos essas concepções são equivocadas, quando não deturpadas.A partir da análise das falas dos profissionais, verificou-se que eles possuem compreensão a algumas características do TEA, no entanto não faziam relação entre essas características e habilidades de brincar. Conclusão: Os profissionais participantes demonstraram pouca segurança em expressar os conceitos básicos do TEA e dificuldades em associar as características do TEA ao brincar funcional. Tal dado pode gerar preocupação quanto a intervenção ofertada a este público. Sugere-se que estudos posteriores investiguem como é realizado o brincar funcional de crianças com TEA.

Palavras-chave: Transtorno do espectro autista, Centro de atendimento psicossocial infanto-juvenil, Pesquisa interdisciplinar.

\section{ABSTRACT}

Objective: To investigate the knowledge of a multidisciplinary team of a Child and Youth Psychosocial Care Center on Autism Spectrum Disorder and to identify the relationship between the characteristics of the Disorder and the child's Play. Method: This was a descriptive, exploratory study with a qualitative approach. The setting for this investigation was a city in the state of Maranhão, the Center for Psychosocial Care for Children and Adolescents (CAPSIJ) was used as a research field. The sample was composed of members of the multidisciplinary team of the CAPS. A questionnaire with open questions was used as data collection material. Results: Study participants, in general, have superficial knowledge about ASD, and, in some

\footnotetext{
${ }^{1}$ Centro Universitário de Ciências e Tecnologia do Maranhão (UniFacema), Caxias - MA.

*E-mail: sannyellerocha17@gmail.com

2Universidade Federal do Maranhão (UFMA), Caxias - MA.

3Universidade Estadual do Maranhão (UEMA), Caxias - MA.
} 
cases, these conceptions are mistaken, when not distorted. From the analysis of the professionals' statements, it was found that they have understanding of some characteristics of TEA, however, did not relate these characteristics and playing skills. Conclusion: The participating professionals showed little confidence in expressing the basic concepts of ASD and difficulties in associating the characteristics of ASD when playing functional. Such data may cause concern regarding the intervention offered to this public. It is suggested that further studies investigate how functional play is performed in children with ASD.

Keywords: Autism spectrum disorder, Child and youth psychosocial service center, Interdisciplinary research.

\section{RESUMEN}

Objetivo: investigar el conocimiento de un equipo multidisciplinario de un centro de atención psicosocial para niños y jóvenes sobre el trastorno del espectro autista e identificar la relación entre las características del trastorno y el juego del niño. Método: Esta fue una investigación exploratoria descriptiva con un enfoque cualitativo. El escenario de esta investigación fue una ciudad en el estado de Maranhão, el campo de investigación utilizado fue el Centro de Atención Psicosocial Infantil (CAPSIJ), la muestra estaba compuesta por miembros del equipo multidisciplinario de los CAPS referidos. Como material de recolección de datos, se utilizó un cuestionario con preguntas abiertas. Resultados: los participantes del estudio generalmente tienen un conocimiento superficial de los TEA y, en algunos casos, estas concepciones son engañosas cuando no están mal representadas. A partir del análisis de las declaraciones de los profesionales, se descubrió que tienen conocimiento de algunas características de los TEA, sin embargo, no tenían relación entre estas características y las habilidades de juego. Conclusión: los profesionales participantes demostraron poca confianza en expresar los conceptos básicos de TEA y las dificultades para asociar las características de TEA con el juego funcional. Dichos datos pueden generar preocupación sobre la intervención ofrecida a este público. Se sugieren estudios adicionales para investigar cómo se realiza el juego funcional de los niños con TEA.

Palabras clave: Trastorno del espectro autista, Centro de atención psicosocial para niños y jóvenes, Investigación interdisciplinaria.

\section{INTRODUÇÃO}

O Transtorno do Espectro Autista (TEA) é classificado como transtorno neurológico que prejudica partes do neurodesenvolvimento, dentre essas regiões afetadas, agrupa-se prejuízos nas atividades como desenvolvimento da motricidade, manipulação, as competências sensoriais, a comunicação e a linguagem, os comportamentos, as competências cognitivas, os afetos e as emoções e por esse motivo podem apresentar déficits na comunicação e nas interações sociais (APA, 2014).

Em pauta da incidência, lamentavelmente no Brasil ainda não existem dados epidemiológicos representativos do país acerca desse índice Paula SC, et al. (2016); Contudo, a última publicação, de 2014, da série de estudos coordenados pelo renomado Center for Disease Control and Prevention (CDC) mostrou uma prevalência de ( 1 a cada 68) crianças nascidas nos Estados Unidos recebem o diagnostico de TEA, (CDC, 2014).

O tratamento da pessoa com TEA deve ocorrer via intervenção multidisciplinar (a depender das necessidades individuais). No âmbito do SUS, este tratamento é oferecido nos CAPS. Deste modo, sabe-se que os Centro de Atenção Psicossocial Infantojuvenil (CAPSi), são as principais instituições públicas de saúde a oferecer atenção a criança e adolescentes que demandem cuidados em saúde mental, e que para melhor atender essa demanda, faz se necessário a presença de equipes aptas e bem preparadas (QUARESMA HMD, 2010; SILVA VG, 2010; QUARESMA IV, 2010).

Esses Centros de atendimento psicossocial, configuram-se suportes essenciais para o acompanhamento de pessoas com transtornos mentais, pois dispõem de acolhimento familiar, visam estimular a integração social, apoio em suas iniciativas e busca por autonomia. Diante disto, Junto ao Sistema Único de Saúde 
(SUS), ambos foram essenciais para a melhoria do atendimento deste, no que se refere ao campo da saúde mental, consolidando os princípios importantes para o atendimento do TEA: a universalização da saúde, a integralidade das ações e a intersetorialidade das políticas (SOUZA RA, 2019; DOS SANTOS JDA, 2019; DA SILVA J, et al., 2019).

Em 2017 e 2018 a triagem (isto é,uma instrumento capaz de auxiliar na detecção de comportamentos atípicos, colaborando no diagnóstico e na percepção de outros sintomas) passou a ser realizada por profissionais de nível superior e os mesmo compunham a equipe multidisciplinar, tais profissionais averiguam e caracterizam o usuário dentro do protocolo de atendimento do CAPSi. Neste processo de investigação a equipe se faz fundamental para que ocorra um diagnostico fidedigno e uma intervenção assertiva. Após analise a equipe participa da decisão dos protocolos de terapias, se será necessário a inserção intensiva, semi-intensiva, não-intensiva ou se encaminha o mesmo a outra instituição (MEDEIRO APA, 2019; TOMÉ CDA, 2019).

Diante disso, o presente trabalho tem como objetivo averiguar os conhecimentos de uma equipe multidisciplinar de um centro de atenção psicossocial infanto-juvenil sobre TEA, através de uma entrevista que foi guiada por um questionário elaborado com perguntas abertas, direcionadas a temática, e, com isso, identificar a relação das características do Transtorno com o Brincar da criança.

\section{MÉTODO}

O estudo tratou-se de uma pesquisa de classe descritiva, exploratória e qualitativa. A pesquisa foi desenvolvida em uma cidade localizada no interior do Maranhão. Para tanto, utilizou-se como campo de pesquisa o Centro de Atendimento Psicossocial do município. Ressalta-se que a escolha do local de pesquisa se deu ao fato de ser referência de atenção ao público de interesse.

Os participantes desta pesquisa foram os integrantes da equipe multiprofissional do CAPS. Considerando consoante, os dados obtidos na secretaria de saúde do município a qual ocorreu a pesquisa, de modo geral, o Centro de Atenção Psicossocial Infanto-Juvenil dispõe de 15 profissionais da saúde, sendo estes: 2 Enfermeiros; 1 Médico; 4 Psicólogos; 1 Terapeuta Ocupacional, 2 Fonoaudiólogos; 1 Técnico de Enfermagem; 2 Pedagogos; 2 Nutricionistas. Utilizou-se como critérios de inclusão do estudo: Ser profissional atuante na Instituição supracitada e aceitar de livre e espontânea vontade participar desta pesquisa. Foram excluídos da pesquisa aqueles que não estiverem em consonância com pelo menos um dos itens acima.

Quanto à coleta de dados, destaca-se que foi aplicado um formulário com perguntas abertas a respeito dos conceitos e características do TEA e com isso, investigar se as características que as pessoas com TEA apresentam pode ter relação com a forma que eles brincam. O formulário foi elaborado com questionamentos que levariam os profissionais a refletirem da importância de se atentar as características comportamentais dessas pessoas e que tais características poderiam potencializar ou prejudicar a forma como elas brincam. Assim, a posse do referido instrumento, ocorreu mediante envelopes individuais, na quantidade exata de profissionais, após a solicitação formal da participação voluntária na pesquisa e assinatura do Termo de Consentimento Livre e Esclarecido.

Ressalta-se que a coleta de dados ocorreu no período de janeiro a fevereiro de 2019 , durante o horário de trabalho dos profissionais e os pesquisadores mantiveram-se à distância durante o procedimento de coleta de dados, para não interferir no processo, nem tampouco intimidar os participantes. Além disso, o sujeito que não pôde responder aos questionamentos naquele dia e/ou momento, o instrumento de coleta de informações não foi entregue, com vistas a manter a fidelidade das respostas

Após a efetivação da coleta de dados, os resultados foram transcritos para análise, interpretação e discussão dos achados. As respostas das entrevistas foram submetidas à análise de Conteúdo, proposta por Bardin (1997), que tem como propósito a compreensão do significado das falas dos sujeitos para além dos limites daquilo que é descrito. Entre as técnicas de Análise de Conteúdo, optou-se pela Análise Temática, que busca os núcleos de sentido, os quais constituem a comunicação e cuja expressão revela algo de importante para o objeto estudado. 
O projeto de pesquisa foi submetido à Plataforma Brasil, e, em seguida, foi direcionado ao Comitê de Ética em Pesquisa (CEP), Sob o parecer número: 97545018.5.0000.5554. Os pesquisadores, responsável e participante, comprometeram-se com as normas preconizadas pela Resolução CNS 466/12 e suas complementares, que tratam dos aspectos éticos da pesquisa envolvendo seres humanos e asseguram que nenhum sujeito será submetido à pesquisa sem ter garantida a sua privacidade e protegida sua integridade física e moral e sem ter assinado o Termo de Consentimento Livre e Esclarecido (TCLE).

O estudo não ofertou nenhum risco previsível. Também não proporcionou benefícios imediatos, mas esperou-se que os resultados encontrados propiciassem aos profissionais atuantes de um CAPS IJ do interior do maranhão a motivação de buscar mais conhecimentos sobre o TEA, o que poderá refletir em uma assistência efetiva a criança com TEA.

\section{RESULTADOS E DISCUSSÃO}

A equipe do CAPS IJ é composta por 14 profissionais os quais trabalham com atendimentos direcionados a crianças e adolescentes com TEA e outras condições. Dessa maneira, todos os membros da equipe foram convidados a participar da pesquisa, entretanto apenas sete profissionais foram incluídos na pesquisa, e o restante excluídos por não aceitação.

Para garantir o sigilo e anonimato dos sujeitos do estudo, seus nomes foram substituído pela primeira letra relacionada a seu cargo profissional acrescido de uma numeração, caso fossem entrevistados mais profissionais de uma mesma categoria, exemplo: (Terapeuta Ocupacional - TO, Enfermeiros - E1, E2, Pedagogo- Pg, Psicólogo - P, Assistente social - AS.

A partir das perguntas abertas, organizou-se os resultados em duas categorias. A primeira categoria relata sobre o conhecimento dos profissionais sobre os conceitos básicos do TEA e a segunda ressalta sobre o conhecimento dos profissionais à cerca das características apresentada no TEA e a relação delas com o Brincar funcional.

$\mathrm{Na}$ Categoria 1 os profissionais foram questionados acerca do que entendiam por TEA. Quanto a isso as respostas obtidas nesta primeira mostram que os participantes do estudo, em geral, possuem conhecimento superficial sobre o TEA, e, em alguns casos essas concepções são equivocadas, quando não deturpadas, conforme pode ser observado nas falas a seguir:

"Não conheço a fundo as características do transtorno em sí". - TO

"É um transtorno do comportamento, que muitas vezes compromete a fala e o desenvolvimento social". - PG

"Transtorno do Espectro Autista é uma alteração do funcionamento da mente que traz prejuízos no desenvolvimento do indivíduo." - P

"Conjunto de sinais e sintomas que incide no comportamento infantil relacionando aspecto cognitivos e afetivos." - E1

"É uma doença, déficit intelectual, não possui cura, possui limitação no desenvolvimento, é dividido por grau, mas que pode se desenvolver." - AS

"Compreendo que pessoas enquadradas no espectro do Autismo terão atraso no desenvolvimento, porém podem se desenvolver, a medida que são assistidas em suas necessidades por uma equipe multidimensional e também pela família. Quando mais cedo acontecer o diagnóstico e as intervenções, melhor serão os resultados." - E2

"Engloba diferentes síndromes marcado por perturbações." - EF

Em vista da pergunta a cerca do conhecimento global que um profissional da área da saúde que trabalha diretamente com pacientes com o transtorno do Espectro Autista (TEA) deveria ter, o participante TO mostra não possuir afinidade com a "patologia", e nem tem conhecimento básico sobre. O que aponta falhas 
voltadas para esse público, e obrigam a indagar, o atendimento de tais pessoas está sendo fidedigno e realmente como deveria ser? Em contrapartida o participante E2 pode de forma sensata elucidar a essência básica do conhecimento sobre o TEA, isso sendo de suma importância, pois um profissional com dimensões básicas sobre o Transtorno teria mais facilidade de colaborar com o desenvolvimento relacionado às suas competências.

Assim Braga TMS e Souza MLR (2013); ressalta que é importante que o profissional conheça e compreenda as peculiaridades do indivíduo, seu histórico familiar. Tal informação poderá orientá-lo quanto às adaptações necessárias como: métodos, currículo e materiais. Para mais, o Transtorno do Espectro Autista (TEA) é relacionado ao desenvolvimento e pode ser identificado por déficits, um estado clinico significativo e persiste na comunicação social e nas interações sociais; déficit nos diálogos verbais e nas expressões não verbais, padrões restringidos e repetitivos de comportamento, interesses e atividades (APA, 2014)

Diante disso as alterações do TEA levam a importantes dificuldades adaptativas e em geral, aparecem antes dos 03 anos de idade, podendo ser percebidas, em alguns casos, já nos primeiros meses de vida. As causas do TEA ainda não estão claramente estabelecidas, entretanto pesquisas apontam que pode ser multifatorial, levando em conta fatores genéticos e ambientais (BJORKLUND G, 2018; SKALNY AV, 2018 e RAHMAN MM, et al., 2018).

Conhecer o perfil do paciente e as características individuais é a base para a programação de um atendimento eficaz. $O$ ensino de habilidades para pessoas com desenvolvimento atípico deve considerar aspectos individuais de aprendizagem, considerando as limitações e investindo em suas potencialidades. Sobre isso, sugere-se que os profissionais conheçam cada caso especificamente para planejar ações que visem o ensino de repertórios importantes para que este indivíduo esteja incluso efetivamente na sociedade e assim, tenham qualidade de vida (SENA RCF, 2015; REINALDE RCF, 2015; E M, et al., 2015).

Nota-se que os profissionais apontam algumas dificuldades coerentes com as reais dificuldade da criança com TEA, pois muitos responderam que afeta a fala, o desenvolvimento social, mas que o mesmo pode se desenvolver com os devidos cuidados. Por outro lado, o EF distorce totalmente da realidade de uma criança com TEA. $O$ que se pressupõe que tais profissionais buscam pouco por informações na literatura sobre o que de certa forma limita $o$ atendimento.

Concordando com isso, os profissionais precisam buscar mais por informações e mudanças, ter um olhar mais flexivo quando se trata do crianças e jovens com TEA, ou seja, adaptar-se de acordo com as necessidades, respeitando suas especificidades, seu tempo e tendo convívio social criando de tal modo mecanismos que faça com que esses aspectos sejam trabalhando da melhor forma possível.

Compreende-se que o conhecimento a respeito das características relacionadas ao TEA parece não está claramente estabelecida frente à algumas respostas emitidas pelos participantes da pesquisa. Em relação a isso, tal fato parece ser preocupante em vista que a Terapia ocupacional é regularmente reconhecida como parte da equipe de prestação de serviços para pessoas com TEA, pois desenvolve principalmente atividades do dia-dia e habilidades sensoriais ou motoras na criança (AUTISMSPEAKS, 2013; VOLKMAR F, 2014; SIEGEL M, 2014; WOODBURY-SMITH M, et al., 2014).

Nesse cenário, Cavalcanti $A$, et al. (2015); articulou que o terapeuta ocupacional é um profissional extremamente importante no acompanhamento de crianças com TEA, pois uma vez que conhece tal prejuízo no comportamento infantil possui um olhar holístico para cada ser humano, levando em consideração suas necessidades, habilidade e tarefas, além disso enxerga o indivíduo como um todo. De acordo com essa deposição, enfatiza-se que tal profissional possui diversos modelos teóricos e abordagens para intervenções na área infantil, incluindo crianças com TEA a partir do momento em que possui domínio e conhecimentos das características de tal.

Além disso, o conhecimento do terapeuta ocupacional sobre as características do TEA é essencial, pois favorece quanto a escolha do melhor plano de ação e atividades a ser desenvolvida com a criança. Para mais, conhecer as características que cada paciente com TEA apresenta, fortalece o vínculo terapêutico e 
com isso aumentará a busca pela afinidade nessa relação "terapeuta-paciente", para que a partir disso se criem e fortaleçam as possibilidades de terapia eficaz, proporcionando então um engajamento e participação mais ativa do paciente (TÁPARO FA, 2011; GIARDINETTO ARSB, 2011).

No âmbitodaspolíticasdesaúde mental, o Assistente Social é o profissional quedesempenha papel relevantena implementação deste tipo política, com isso as questões que envolvem os direitos sociais do público infantil, incluído o TEA fica sobe função central do referido profissional, assumindo assim grande relevância nas funções centrais de realização das ações em prol do melhoramento do quadro clinico apresentado pela criança. Tal dado pode ser preocupante à medida que os profissionais não apresentam total domínio do que se refere os conceitos de TEA, tendo em vista o papel do AS nos programas e projetos sociais destinada a melhoria na sociedade de pessoas com esse diagnóstico (NIETSCHE AD, 2011).

Para mais, as falas dos interlocutores levantam reflexão sobre sua prática de assistência voltada para esta população, uma vez que o TEA é um grupo de transtornos que proporciona especificidades como (comprometimento no desenvolvimento social, no comportamento e dificuldades de comunicação e outros) que merecem atenção bem especificas, (American Psychiatric Association, 2013), pois para realizar uma assistência bem efetiva faz-se necessário um conhecimento bem fundamentado.

$\mathrm{Na}$ categoria 2 abordou-se aos participantes do estudo questões no que diz respeito das característicasdo TEA e a relação delas com o brincar da criança, em relação a isso os participantes revelaram que essas características seriam:

"Déficit intelectual, possui limitações na fala verbal e não verbal, inquietação, movimento repetitivo, possui sensibilidade sensorial, não possui figura de linguagem." - AS

"As principais características referem-se à socialização das crianças sentido no isolamento, escolalias, balanço corporal, pouca afetividade, interesse por coisas que outras crianças não tem." - E1

"Dificuldades no relacionamentos social, em alguns casos podem apresentar ecololia, hipersensibilidade autitivo, dificuldade no cognical, motricidade, na simbolização." - P

"Não tem contato visual, Dificuldade de socializar, Estereotipias, dificuldade na fala, anda na ponta dos pés, empilha objetos, fala robótica". - PG

"Atraso na fala, dificuldade na socialização, pouco contato visual, ecolalias, estereotípicas." - TO

"Corália, estereotipia, repetição de palavras, choro e contato visual." - EF

"Atraso de desenvolvimento, dificuldade na fala, estereotipias, ecolalias, catatonia, dificuldade de abstração, dificuldade de olhar nos olhos e manter o olhar, intolerância a alguns ruídos, o que, por vezes, o leva ao isolamento." - E2

A partir da análise das falas dos profissionais, verifica-se que eles possuem compreensão a algumas características do TEA, no entanto não fazem relação entre essas características e habilidades de brincar. Para (APA, 2014) as manifestações comportamentais mais frequentes no TEA incluem comprometimentos no desenvolvimento sociocomunicativo, bem como a presença de comportamentos estereotipados e de um repertório restrito de interesses e atividades, sendo que os sintomas nessas áreas, quando tomados conjuntamente, limitam ou dificultar o funcionamento diário da criança. $O$ ato da brincadeira em si exige habilidades de interação social, seguimento de instruções, seguimento de regras, imaginação, contato visual dentre outras características, e isso implica no funcionamento da criança com TEA em brincadeiras.

No que diz respeito a característica supracitada de que crianças com TEA apresentam dificuldades em afeto e contato visual. Locatelli PB e Santos MFR (2016); relata que no autismo existe uma dificuldade de perceber sutileza e linguagem, de decifrar gestos, movimentos e intenções nas expressões faciais das 
outras pessoas. $\mathrm{O}$ autor ainda aponta que pessoas com TEA interpretam tudo de forma literal. Destaca que uma característica mais integrante do autista, nos mais variados aspectos, seja a questão do olhar, para demonstrar $o$ afeto usa-se vários canais, exemplificando toque físico, tato visual, e que o autista não tem essas habilidades, não gosta do contato, seja físico ou visual, pois lhes proporciona um enorme desconforto.

Além disso, o TEA proporciona déficits na comunicação não verbal e verbal, contribuindo então a desenvolver prejuízos na linguagem e na fala. Para (CONTI-RAMSDEN G, 2013; MOK PL, 2013; PICKLES A, et al., 2013) crianças com atraso no desenvolvimento são mais vulneráveis a dificuldades na fala e no processo de linguagem quando comparadas as crianças em desenvolvimento típico.

Essas dificuldades comprometem a forma de brincar que a criança apresenta, tendo em vista que a brincadeira exige uso da imaginação, compreensão de instruções orais, as vezes é necessário permanecer sentado ou em pé por tempo programado, esperar a vez, fazer alternância de turno, além disso envolve as habilidades de funções executiva (um conjunto de habilidades cognitivas necessárias para realizar diversas atividades que demandam planejamento e monitoramento de comportamentos intencionais relacionados a um objetivo ou a demandas ambientais), essas habilidades são importantes para a realização de brincadeiras e tarefas (OMARI C, 2011; VALIATI MRMS 2011).

Segundo um caso com crianças diagnosticadas com TEA, conclui que com estratégias e intervenções feitas dentro de 10 meses mostraram-se positivas para o desenvolvimento contínuo, esse desenvolvimento estava relacionado a conclusão de que o contato visual, atenção compartilhada e imitação estão entre as funções básicas que podem ser desenvolvidas, com intervenções adequadas. As intervenções utilizadas nesse estudo foram Identificação de partes do corpo nela mesma, Identificação de cores, Imitação verbal, Imitação motora sem objetos, Identificação de pessoas, Encaixe de formas, Identificação de objetos e Brincar funcional (CAMINHA VL, 2016; HUGUENIN J Y, 2016; ASSIS LMD, et al., 2016).

Dessa forma, o brincar funcional é fundamental para o desenvolvimento, e a compreensão dessa premissa é importante para que seja trabalhada não como uma brincadeira simples e sim como uma intervenção que seja aplicada de forma seria e assistida pelos profissionais e se estenda aí berço familiar. A informação é um ponto crucial para o desenvolvimento e cabe de forma individual aos profissionais terem a responsabilidade de ir a buscar das informações devidas para qualificar ainda mais o atendimento e ter mais eficácia em seus objetivos.

\section{CONCLUSÃO}

Os resultados deste estudo apontam que os profissionais participantes demonstram pouca segurança em expressar os conceitos básicos do TEA, este comportamento pode gerar preocupação, uma vez que o atendimento ofertado pelos CAPS IJ alcança muitas famílias que buscam por uma melhor qualidade de vida e desenvolvimento da criança com esse diagnostico. E, conhecer, sobretudo as características básicas do Transtorno, é fundamental para um atendimento mais eficiente na prática de assistência voltada para esta população. O estudo aponta também que, embora alguns participantes conheçam certas características das crianças com TEA, encontraram dificuldades em associar elas ao brincar funcional. Sugere-se que estudos posteriores investiguem como é realizado o brincar funcional de crianças com TEA.

\section{REFERÊNCIAS}

1. AMERICAN PSYCHIATRIC ASSOCIATION - APA. Manual diagnóstico e estatístico de transtornos mentais: DSM-5. Associação Brasileira de Psiquiatria. Trad. Maria Inês Corrêa Nascimento. 5 ed. Porto Alegre: Artmed, 2014.

2. AMERICAN PSYCHIATRIC ASSOCIATION - APA. Diagnostic and statistical manual of mental disorders (DSM$\left.5^{\circledR}\right)$. Arlington, VA: American Psychiatric Publishing, 2013.

3. AUTISM SPEAKS. (2013). 2013 national conference- Conference schedule.

4. BRAGA TMS e SOUZA MLR. Adaptação de recursos para aluno com DV na Educação infantil: relato de experiência. In: Congresso Brasileiro de Educação Especial, III., 2008, Universidade Federal de São Carlos. Anais eletrônicos. São Carlos: UFSCar, 2013.

5. BJØRKLUND G, et al. Toxic metal(loid)-based pollutants and their possible role in autism spectrum disorder. Revista Environmental Research, 2018; 166: 234-25. 
6. CAVALCANTI A, et al. Estrutura da prática da Terapia Ocupacional: domínio \& processo. Revista de Terapia Ocupacional da Universidade São Paulo, São Paulo, 2015; 26: 1-49.

7. CENTER FOR DISEASE CONTROL AND PREVENTION - CDC. Diagnostic and statistical manual of mental disorders. 2014.

8. CONTI-RAMSDEN G, et al. Adolescents with a history of specific language impairment (SLI): Strenghts and difficulties in social, emotional and behavioral functioning. Revista Elsevier, 2013; 34 (11): 4161-4169.

9. LOCATELLI PB e SANTOS MFR. AUTISMO: Propostas de Intervenção. Revista Transformar, 2016; 8(8), $203-220$.

10. MEDEIRO APA e TOMÉ CDA. Desafios para efetivação da política de atenção à saúde mental no Centro de Atenção Psicossocial Infantojuvenil (CAPSi) de Patos/PB. Revista repositório digital, 2018; 1-14.

11. NIETSCHE AD. Estudos Sobre Autismo Na Perspectiva Dos Direitos. Trabalho de Conclusão de Curso em Serviço Social. Universidade Federal de Santa Catarina. Florianópolis, 2011.

12. OMARI C e VALIATI MRMS. A Terapia Ocupacional na Pediatria. In: VALIATI M.R.M.S et al. Desenvolvimento da criança e do adolescente. Curitiba: Íthala, 2011. p. 75-82.

13. PAULA CS, et al. Estudantes de Psicologia Concluem a Graduação com Uma Boa Formação em Autismo? Revista Psicologia - Teoria e Prática, 2016; 18(1)

14. QUARESMA HMD, et al. Autismo infantil: concepções e práticas psicológicas. Revista Brasileira de Ciência da Saúde, São Caetano do Sul, 2010; 14(4): 85-90.

15. SENA RCF RCF, et al. Practice and know ledge of nurses about child autism. Revista de Pesquisa: Cuidado é Fundamental Online. 2015; 2915; 7(3): 2707.

16. SOUZA RA, et al. Uma reflexão sobre as políticas de atendimento para as pessoas com transtorno do espectro autista. Revista Cadernos UniFOA, 2019; 40: 95-105.

17. TÁPARO FA e GIARDINETTO ARSB. Avaliação das atividades realizadas em uma instituição de atendimento de crianças e jovens com autismo: contribuições com a implantação de um serviço de terapia ocupacional. Marília: Unesp, 2011.

18. VOLKMAR F, et al. Practice parameter for the assessment and treatment of children and adolescents with autism spectrum disorder. Journal of the American Academy of Child \& Adolescent Psychiatry. 2014; 53(2), $237-257$.

19. CAMINHA VL, et al. Autismo: vivências e caminhos. São Paulo: Editora Edgard Blücher Ltda. $2016 ; 11$. 